\title{
APPLICATION OF ARTIFICIAL NEURAL NETWORKS TO FORECASTING MONTHLY RAINFALL ONE YEAR IN ADVANCE FOR LOCATIONS WITHIN THE MURRAY DARLING BASIN, AUSTRALIA
}

\author{
JOHN ABBOT ${ }^{1,2,3} \&$ JENNIFER MAROHASY ${ }^{1,2}$ \\ ${ }^{1}$ Climate Modelling Laboratory, Noosa, Queensland, Australia. \\ ${ }^{2}$ Institute of Public Affairs, Melbourne, Victoria, Australia. \\ ${ }^{3}$ James Cook University, Townsville, Queensland, Australia.
}

\begin{abstract}
Much of Australia regularly experiences extremes of drought and flooding, with high variability in rainfall in many regions of the continent. Development of reliable and accurate medium-term rainfall forecasts is important, particularly for agriculture. Monthly rainfall forecasts 12 months in advance were made with artificial neural networks (ANNs), a form of artificial intelligence, for the locations of Bathurst Deniliquin, and Miles, which are agricultural hubs in the Murray Darling Basin, in southeastern Australia. Two different approaches were used for the optimisation of the ANN models. In the first, all months in each calendar year were optimised together, while in the second approach, rainfall forecasts for each month of the year were made individually. For each of the three locations for most months, higher forecast skill scores were achieved using single-month optimizations. In the case of Bathurst, however, for the months of November and December, the root mean square error (RMSE) for all-month optimisation was lower than for single-month optimisation. The best overall rainfall forecasts for each site were obtained by generating a composite of the two approaches, selecting the forecast for each month with the lowest forecast errors. Composite model skill score levels of at least $40 \%$ above that of climatology were achieved for all three locations, whereas skill level derived from forecasts using general circulation models is generally only comparable to climatology at the long-lead time of 8 months.
\end{abstract}

Keywords: artificial neural network, flooding, forecast, Murray Darling Basin, rainfall, skill score.

\section{INTRODUCTION}

The Murray Darling Basin (MDB) covers 1,059,000 square kilometres, or 14\% of Australia's land area, and contains a large proportion $(65 \%)$ of the irrigated land. The MDB accounts for more than half of Australia's total water use, of which more than $80 \%$ is used by agricultural industries [1], generating nearly $40 \%$ of the value of agricultural production of Australia. Over the past several decades, competing requirements of agriculture and the environment have led to difficulties in formulating water policies for the region $[2,3]$. This issue is exacerbated because the natural climate of the region is characterized by periods of extreme drought, followed by periods of heavy rainfall [4]. It would be beneficial for both policy makers and farmers if more accurate medium-term rainfall forecasts were available, particularly at long lead times [5-8]. However, most attempts to forecast rainfall with long lead times have resulted in models with poor predictive skill $[9,10]$ often only comparable to, or below, climatology.

Two principle approaches have been applied to medium-term rainfall forecasting: statistical models [11, 12] and general circulation models (GCMs) [13]. These approaches have been more commonly used to produce seasonal forecasts, and less extensively for monthly rainfall forecasts. Statistical models typically use a set of lagged input variables to compute desired output rainfall, usually large-scale climate variables or indices [14-17]. 
Artificial neural networks (ANN), a form of machine learning, can be classified as a type of statistical model that offers several important advantages over the more simple statistical models traditionally used. ANNs have the capacity to accommodate non-linear relationships, and the flexibility in testing multiple inputs, particularly where their influences may be due to combinations of drivers that interact in poorly understood ways. Preliminary research suggests ANNs have the potential to produce skilful seasonal and monthly rainfall forecasts in many parts of the world [18, 19] including Australia [20-23]. Forecasts from GCMs, however, are used to produce the operational medium-term rainfall forecasts issued by the Australian Bureau of Meteorology (BOM), despite evidence that these forecasts are typically less skilful, and despite substantial efforts to enhance performance over many years [24, 25].

Currently operational medium-term seasonal rainfall forecasts issued by the BOM use output from a GCM, the Predictive Ocean Atmosphere Model for Australia (POAMA) [10, 13]. Prior to May 2013, operational rainfall forecasts generated by the BOM were produced by simple statistical models that used only four input values for the entire continent. These inputs were derived from two lagged sea surface temperatures, SST1 and SST2 [11, 12] relating to the Pacific and Indian oceans respectively. Both of these sea surface temperature model inputs were lagged by one and three months. In reviewing the performance of this simple statistical model, Fawcett and Stone [12] described the skill level demonstrated for seasonal rainfall forecasting as "only moderate, better than climatology or randomly guessed forecasts".

Risbey et al. [16] examined concurrent relationships between seasonal rainfall over Australia with individual ENSO-related drivers additional to Southern Oscillation Index (SOI), including the ENSO Modokai Index (EMI) Niño 3, Niño 3.4 and Niño 4, which are all measures of sea surface temperature (SST) difference in the equatorial Pacific. Other non-ENSO indices considered were the Indian Ocean Dipole (DMI), the Southern Annular Mode (SAM) and the Madden-Jullian Oscillation (MJO). Risby et al. [16] found that analysis based on linear correlations between rainfall and individual drivers could only generate crude indications of the relative importance of individual climate indices, and did not account for possible interactions. Nevertheless, their results indicate which individual driver might be more significant for determination of seasonal rainfall throughout Australia. SOI was most the influential in northern parts of Queensland. For south east Queensland, the identity of the dominant index was more variable, and depended on the season. Many other studies have reported on the relationships of ENSO-related indices to rainfall in eastern Australia [15, 26-28]. There is also evidence that the relationship between ENSO and rainfall is modulated by the phases of the Inter-decadal Pacific Oscillation (IPO) [29-31] and when the IPO is in a negative phase, the impact of ENSO on rainfall is enhanced [32].

A strong concurrent relationship between rainfall and a particular input variable does not necessarily translate into a strong lagged relationship [14]. It is lagged relationships that are essential for forecasting. Chiew et al. [33] examined linear correlations between rainfall and ENSO indices (SOI and SSTs) for the Queensland region, including lagged relationships. The strongest linear correlations, $r$ above 0.4 , were found for spring rainfall. The geographical coverage and intensity of the strongest correlations declined as lags were progressively increased from 0 to 1, 2 and 3 months. Correlations with summer rainfall were less expansive geographically, and these also diminished with lag time. Kirono et al. [34] investigated linear correlations between 12 individual climate indices, lagged for 1 or 2 months, and seasonal rainfall across Australia including SOI, Niño 3, Niño 4, DMI, SAM. Results for south-east Queensland exhibited the sporadic influence of individual lagged climate indices. 
Schepen et al. [14] estimated the influence of 13 individual lagged climate indices on seasonal rainfall for geographical grid areas across Australia. The indices studied included SOI, Niño 3, Niño 3.4, Niño 4, DMI, EMI, and also the Indian Ocean East Pole index (EPI) at lag periods of 1, 2 and 3 months. The variability in the strengths of relationships between lagged climate indices, and rainfall was calculated by using the pseudo-Bayes factor that can accommodate non-linear relationships. This factor is probably more useful in providing a measure of the strength of rainfall-input relationships than the Pearson correlation coefficient (r). However, evaluations using the pseudo-Bayes factor are still potentially limited in that they evaluate each rainfall-input relationship in isolation from the effect of other inputs. This may not give a true indication of a specific input's influence when introduced in combinations with other inputs.

This study builds on a previous investigation using ANNs [35] where monthly rainfall forecasts were made for Miles in Queensland with lead times between 1 and 9 months. In this study, two additional sites in the Murray Darling are considered, Bathurst and Deniliquin, both in New South Wales. This study also presents results for optimisation of ANNs for individual months, whereas the previous study was limited to optimisation of all months together.

\section{DATA AND METHODS}

The three stations forecasted for in this study included Bathurst Agricultural Station, Denilquin, and Miles Post Office all of which began collecting data in 1908, 1858, and 1885 (respectively). Each site is also in close proximity to a $250 \mathrm{~km} \times 250 \mathrm{~km}$ grid area where comparative monthly forecasts have been published using the GCM POAMA [10].

Each location is still operating as a weather station today, and data from these stations located within the Murray Darling Basin (Fig. 1) was used in the ANNs.

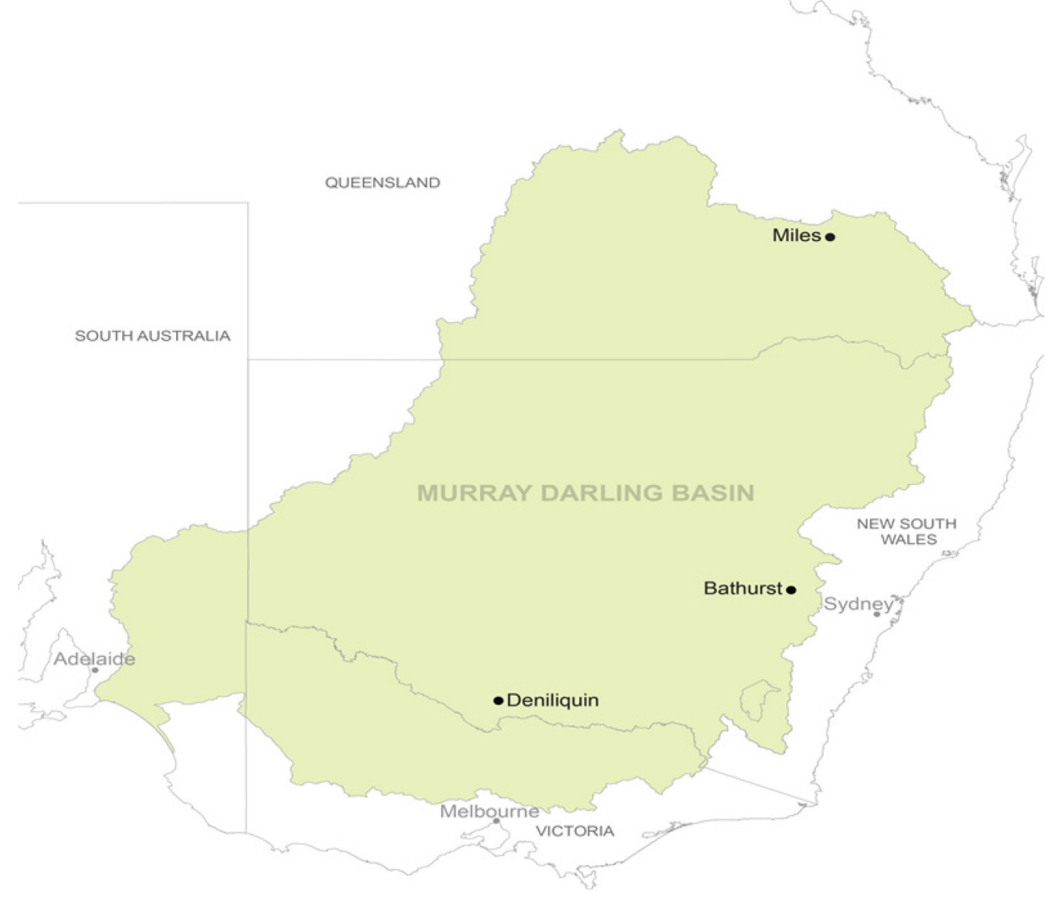

Figure 1: Map showing locations of Bathurst, Deniliquin and Miles within the Murray Darling Basin. 
Local minimum and maximum temperatures and a set of climate indices were input as monthly values to the ANNs. Previous investigations have shown that local temperatures and rainfall contribute as significant inputs to monthly rainfall forecasts for eastern Australia [20]. The climate indices used as inputs were the SOI, DMI, Niño 4, Niño 3.4, Niño 3, Niño 1.2 and the IPO. As discussed above, each of these has been shown to have potential value as a predictor of future rainfall over eastern Australia, and because monthly values are available over a long historical period extending back as far as the observational rainfall records considered. Other climate indices that may be influential in the prediction of rainfall in south-east Australia were not used as inputs in this study because their availability is restricted to a shorter time period. For example, monthly values of the SAM are only available from the Climate Explorer website from 1957 onwards, whereas the other inputs used extend back for more than a century. The forecasts were run with the full set of inputs, and also local rainfall, with each lagged up to 12 months.

The total input data sets of monthly data used comprised: Bathurst (July 1909 to June 2014); Deniliquin (January 1877 to June 2014); Miles (January 1888 to July 2011), (Table 1). The dataset considered for Miles was taken to mid-2011 because of gaps in the corresponding temperature series. For each site, the data were divided into training (75\%), evaluation (15\%) and test sets $(10 \%)$. Test data sets used in training were not used in testing.

Values for DMI and the four Niño indices were sourced from the Royal Netherlands Meteorological Institute Climate Explorer - a web application that is part of the World Meteorological Organisation and European Climate Assessment and Dataset project. Values for IPO were provided by the UK Met Office. Values of SOI, and also local minimum and maximum temperatures, were obtained from from the Australian Bureau of Meteorology.

All of the above climate indices with local rainfall and temperatures were provided as input to Neurosolutions Infinity software and used to build ANN models. Many different architectures of ANNs have been used to make forecasts of rainfall [18-23]. A common approach in the selection of an ANN architecture is through simple trial and error of candidate models [20-23] using a limited set of input data, and select the ANN that produced the minimum error. This model is then applied in the investigation with all the data input sets. This selection process can be a very time-consuming. The recently available software Neurosolutions Infinity gives access to many different ANN architectures, but has the facil-

Table 1: Initial input data sets used for ANN models and sources.

\begin{tabular}{lll}
\hline Input & Lags (months) & Source \\
\hline Rainfall & $12-24$ & Australian BOM \\
Maximum Temperature & $12-24$ & Australian BOM \\
Minimum Temperature & $12-24$ & Australian BOM \\
SOI & $12-24$ & Australian BOM \\
IPO & $12-24$ & UK Met Office \\
DMI & $12-24$ & Climate Explorer \\
Nino 1.2 & $12-24$ & Climate Explorer \\
Nino 3 & $12-24$ & Climate Explorer \\
Nino 4 & $12-24$ & Climate Explorer \\
Nino 3,4 & $12-24$ & Climate Explorer \\
\hline
\end{tabular}


ity to automate both selections of ANN architecture and input data. This offers a great advantage in terms of arriving at an optimum ANN configuration for every data set of interest without a prohibitive time outlay. The Infinity program uses a pre-set formula incorporating RMSE, mean absolute error (MAE) and correlation coefficient (r) to evaluate the accuracy for each neural network model and a corresponding set of selected inputs tested. Based on this formula, the program determines which ANN model and set of inputs is optimal. For the datasets used in this investigation, the optimal ANN configuration automatically selected by Neurosolutions Infinity was a probabilistic neural network (PNN). A PNN has four layers, an input layer, one hidden layer, a summation layer and an output layer. Each attribute considered was lagged up to 12 months. The data were divided into training (75\%), evaluation (15\%) and test sets (10\%). RMSE and Pearson correlation coefficients are used to compare the skill of rainfall forecasts generated by the ANNs, POAMA and also climatology [20,36].

Two approaches were used for ANN optimization. With the first approach, designated as "all-month optimization", data for all 12 months of the year was included as input and optimised together, as in our previous studies $[10,11]$. With the second approach, designated as "single-month optimisation", forecasts corresponding to each calendar month were performed individually, so that 12 optimisations were carried out to produce forecasts for the entire year.

Equation (1) was applied to give a forecast skill score relative to climatology:

$$
\text { Skill score }=\frac{[\text { RMSE }(\text { climatology })-\text { RMSE }(\text { model })]}{\text { RMSE }(\text { climatology })} * 100 \%
$$

This is analogous to an equation used in studies where a skill score has been calculated for forecasts based on POAMA [10]. Applying this equation, it follows that if the calculated values of RMSE from climatology and for a particular model are equal, the forecast skill score will be zero. For a perfect model forecast, the RMSE for the model will be zero, and the calculated skill score will be $100 \%$. Any negative values calculated from eqn (1) indicate a forecast skill score below that of climatology.

\section{RESULTS AND DISCUSSION}

Optimizations performed with Neurosolutions Infinity software to generate forecasts at the three sites produced optimised models regarding both the neural network architecture and the associated inputs. In each case, there were initially 120 lagged input variables available to relate to each required output rainfall value (Table 1). In all cases, the fraction of these input retained by the Infinity software in the optimised model was a small subset of the initial input set made available, typically only $5 \%$ to $10 \%$. This is illustrated for single-month optimisations for Deniliquin in Tables 2 and 3, where each numerical entry corresponds to the lag period for the particular retained attribute.

RMSE values were calculated corresponding to each month of the year for test data using (i) all-month optimisation and (ii) single-month optimisation for Bathurst (January 2005 to June 2014), Deniliquin (July 2004 to June 2014) and Miles (August 2004 to July 2011) respectively (Table 4). Inspection of these values shows that for each location, for the majority of months, higher skill was achieved using the single-month optimisation. Only in the case of Bathurst, for the forecast months of November and December did the all-month method produces lower errors, while for Miles and Deniliquin the single-month optimisations produced lower errors for all 12 calendar months. 
Table 2: Retained input data for optimal neural networks for Deniliquin for single-month optimizations (January to June).

\begin{tabular}{|c|c|c|c|c|c|c|}
\hline Input & Jan & Feb & Mar & Apr & May & Jun \\
\hline Rainfall & & & & 12 & 12 & \\
\hline $\operatorname{Max} \mathrm{T}$ & $20,22,24$ & $19,20,23$ & & & 14,17 & $\begin{array}{l}12,13,16,18, \\
21,23\end{array}$ \\
\hline Min T & 19 & 17 & $12,22,23$ & & & \\
\hline SOI & & $15,17,18,20$ & & & & \\
\hline IPO & 19 & & $13,15,18$ & & & \\
\hline DMI & & & $13,23,24$ & & & \\
\hline Nino 1.2 & & $13,14,24$ & & & & \\
\hline Nino 3 & & 12 & & & & \\
\hline Nino 4 & & & 13 & & & \\
\hline Nino 3.4 & & & & $12,13,14$ & & \\
\hline
\end{tabular}

Table 3: Retained input data for optimal neural networks for Deniliquin for single-month optimizations (July to December).

\begin{tabular}{lllllll}
\hline Input & Jul & Aug & Sep & Oct & Nov & Dec \\
\hline Rainfall & & & & & & 21 \\
Max T & $12,13,19$ & & & 16 & $19,20,24$ & \\
Min T & 21 & 16 & $13,15,21$ & $16,18,24$ & 15 & \\
SOI & & 12 & & $17,19,20$ & & 13,14 \\
IPO & & 12 & & $15,19,21$ & 13,23 \\
DMI & & & 13,15 & & \\
Nino 1.2 & & 16 & & $14,21,22$ & $13,21,24$ \\
Nino 3 & 23 & 24 & 14 & 16,19 & 14,16 \\
Nino 4 & & & & 21 & \\
Nino 3.4 & & 12 & 14 & & \\
\hline
\end{tabular}

Composite forecasts were constructed by taking the best forecasts for each month from both methods. Therefore, composite and single month forecasts for Miles and Deniliquin are equivalent, whereas for Bathurst the composite forecast incorporates two months from the all-month optimisation.

Values of forecast skill were calculated using eqn (1) for Bathurst, Deniliquin and Miles corresponding to the composite forecasts (Table 5). Skill scores were above climatology for all forecast months at each of the three locations. Average skill levels, relative to climatology, in the range 42-48\% are achievable for rainfall forecasts with 12 month lead time. Table 6 shows the corresponding Pearson correlation coefficients between observed and ANN forecast rainfall are in the range 0.80 to 0.98 . 
Table 4: Root mean square errors for monthly rainfall forecasts for Bathurst, Deniliquin and Miles using all-month and single month optimisation.

\begin{tabular}{lllllll}
\hline & \multicolumn{3}{c}{ All-month RMSE (mms) } & \multicolumn{3}{c}{ Single-month RMSE (mms) } \\
\cline { 2 - 6 } Month & Bathurst & Deniliquin & Miles & Bathurst & Deniliquin & Miles \\
\hline January & 27.0 & 13.8 & 37.9 & 11.4 & 11.8 & 31.5 \\
February & 23.7 & 28.1 & 43.8 & 19.5 & 9.4 & 28.8 \\
March & 34.2 & 30.8 & 35.5 & 31.1 & 7.8 & 30.3 \\
April & 21.4 & 16.3 & 24.3 & 12.5 & 12.2 & 14.2 \\
May & 22.5 & 19.5 & 27.1 & 7.9 & 13.2 & 15.2 \\
June & 25.8 & 17.3 & 57.6 & 15.7 & 20.1 & 28.7 \\
July & 19.5 & 11.5 & 21.8 & 13.1 & 7.5 & 11.8 \\
August & 21.3 & 20.0 & 29.4 & 15.5 & 9.4 & 19.0 \\
September & 35.5 & 17.1 & 28.2 & 18.5 & 14.4 & 12.6 \\
October & 24.5 & 21.1 & 28.2 & 22.4 & 11.2 & 16.7 \\
November & 36.2 & 16.1 & 33.7 & 47.9 & 16.8 & 13.0 \\
December & 36.2 & 17.0 & 55.7 & 38.7 & 9.3 & 44.8 \\
\hline
\end{tabular}

Table 5: Forecast skill score (\%) for rainfall forecasts at 12 months lead for Bathurst, Deniliquin and Miles using a neural network.

\begin{tabular}{llll}
\hline Month & Bathurst & Deniliquin & Miles \\
\hline January & 70.4 & 35.9 & 42.3 \\
February & 50.8 & 71.3 & 33.2 \\
March & 41.5 & 80.6 & 42.9 \\
April & 59.2 & 22.9 & 35.1 \\
May & 60.7 & 43.3 & 37.6 \\
June & 29.3 & 57.4 & 45.4 \\
July & 36.4 & 17.9 & 53.8 \\
August & 56.3 & 53.4 & 34.6 \\
September & 44.6 & 18.7 & 62.3 \\
October & 39.2 & 66.3 & 50.6 \\
November & 42.8 & 35.4 & 31.8 \\
December & 45.4 & 67.8 & 49.0 \\
\hline
\end{tabular}

Figures 2, 3 and 4 show plots of observed and forecast monthly rainfall for each site for the composite for Bathurst, Deniliquin and Miles respectively. In each case the test period is shown which corresponds to $10 \%$ of the total rainfall period used in the ANN model. Of particular interest is the prominent forecast peak in January 2010/January 2011 for Miles in Fig. 4, when extremely heavy rainfall was experienced in Queensland. 
Table 6: Correlation coefficients for rainfall forecasts at 12 months lead for Bathurst, Deniliquin and Miles using a neural network.

\begin{tabular}{llll}
\hline Month & Bathurst & Deniliquin & Miles \\
\hline January & 0.96 & 0.96 & 0.94 \\
February & 0.92 & 0.97 & 0.80 \\
March & 0.89 & 0.98 & 0.90 \\
April & 0.92 & 0.91 & 0.83 \\
May & 0.97 & 0.91 & 0.94 \\
June & 0.84 & 0.94 & 0.87 \\
July & 0.94 & 0.94 & 0.72 \\
August & 0.98 & 0.95 & 0.80 \\
September & 0.96 & 0.94 & 0.96 \\
October & 0.77 & 0.98 & 0.91 \\
November & 0.89 & 0.84 & 0.90 \\
December & 0.99 & 0.98 & 0.88 \\
\hline
\end{tabular}

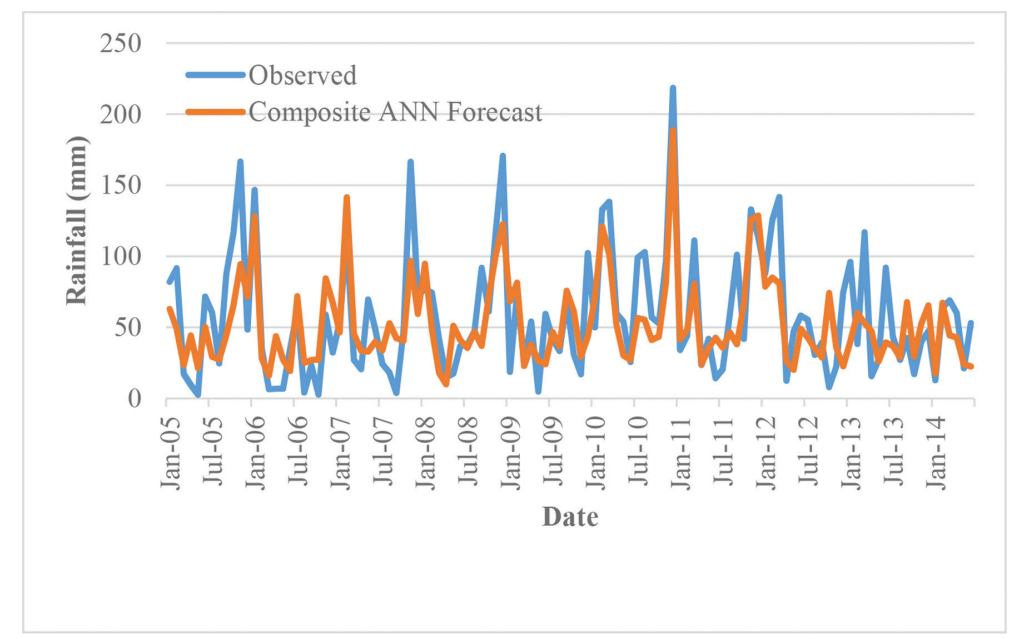

Figure 2: Forecast and observed monthly rainfall for Bathurst using the composite neural network. Test period January 2005 to June 2014.

In previous reported studies using ANNs to forecast monthly rainfall in Queensland [2023], the approach used was to optimise forecasts for the 12 calendar months together, for a given set of inputs. This approach consistently produced results with better skill than either climatology or POAMA. However, the present study shows that the monthly rainfall forecast skill can in most instances be improved when individual months are optimised. This is because single-month optimization enables the variability in the influence of specified lagged climate indices at different times during the year to be considered [14]. 


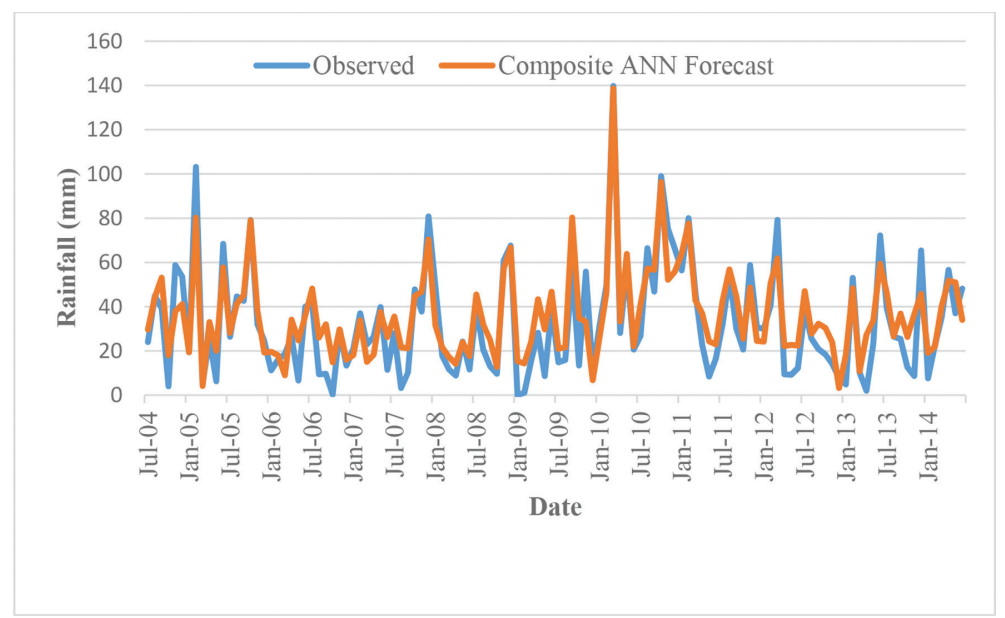

Figure 3: Forecast and observed monthly rainfall for Deniliquin using the composite neural network. Test period July 2004 to June 2014.

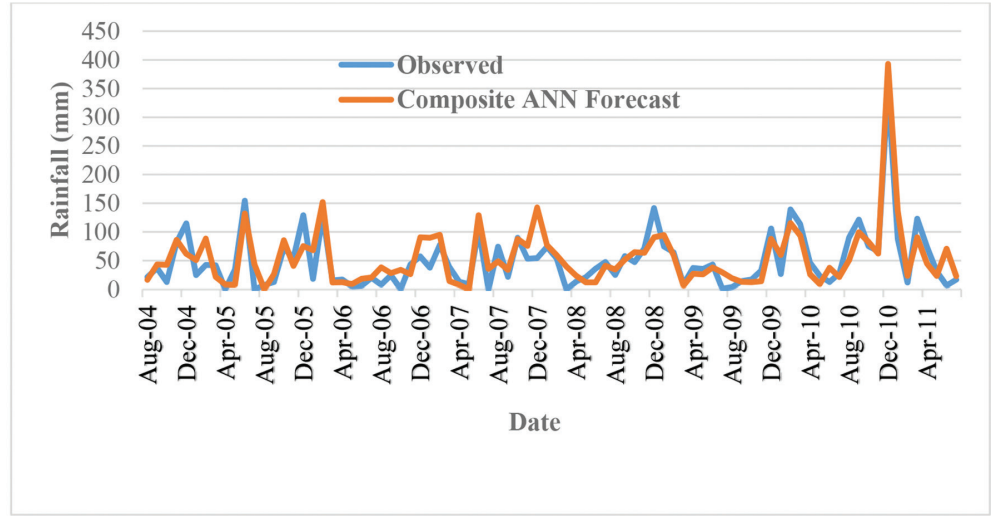

Figure 4: Forecast and observed monthly rainfall for Miles using the composite neural network. Test period August 2004 to July 2011.

Ideally, the output from the ANNs would be compared directly with output from POAMA, using identical lead times, locations and test periods. However, only very limited results from POAMA have been made available by the BOM to enable comparative skill analysis. Comparisons based on the limited data directly provided by the BOM, and also published studies, show that the ANN method has superior skill for monthly rainfall forecasting in the Murray Darling Basin region of Australia at the long lead times of 12 months. Table 7 shows a comparison of results using a lead time of 12 months from this study using an ANN, with results from POAMA at a lead time of 8 months. These results from POAMA corresponded to the longest lead time available in the published literature. Bearing in mind that results from POAMA generally decrease with longer lead times, so the comparison made is not biased against the GCM. In particular, Table 7 shows that applying the composite ANN approach gives skill levels above climatology for all 12 months of the year, with values between $22 \%$ and $69 \%$. In contrast, for forecasts derived from POAMA, results for 9 months of the year 
Table 7: Forecast skill as a percentage for monthly rainfall forecasts for Miles with reference to climatology for the composite ANN model and POAMA. The test period was August 2004 to December 2010.

\begin{tabular}{llc}
\hline Month & ANN (lead 12 months) & POAMA (lead 8 months) \\
\hline January & 30.2 & 6.0 \\
February & 22.2 & -1.7 \\
March & 49.3 & -6.3 \\
April & 39.3 & -40.6 \\
May & 33.1 & -5.9 \\
June & 43.3 & -1.2 \\
July & 62.1 & -3.4 \\
August & 30.6 & 0.0 \\
September & 69.0 & -2.0 \\
October & 43.0 & -8.0 \\
November & 22.0 & 8.1 \\
December & 48.6 & -16.1 \\
\hline
\end{tabular}

have skill levels at, or below, climatology, and results for 4 months have skill between 0 and $5 \%$, with no results above $10 \%$. Taking the averages over 12 months, the results from POAMA have a skill level of $-3.9 \%$ with reference to climatology, while the average for results from the composite neural network is $42.5 \%$.

The measure of the forecast skill score expressed in eqn (1) is analogous to the formula applied by Hawthorne et al. [10] to evaluate monthly rainfall forecast skill using POAMA. In that study, results for various lead times and $250 \mathrm{~km} \times 250 \mathrm{~km}$ grid regions in Australia were presented along a skill spectrum with reference to climatology based on RMSE, extending between $-20 \%$ and $20 \%$. To facilitate quantitative forecast skill comparisons, those published results have been translated into numerical ranges of values (Table 8). This gives the number of calendar months of the year where the POAMA forecast skill falls into particular ranges. Three geographical grid locations are considered, identified as H, E and G [10] as these are in the geographical proximity of the three locations Bathurst, Deniliquin and Miles, respectively.

Using the composite ANN model for Bathurst, all 12 forecast months showed a forecast skill above climatology, with skill levels in the range $24 \%$ to $70 \%$. The results from POAMA for grid region $\mathrm{H}$ at 6 and 8 month lead, gave 7 and 8 months, respectively, with skill levels below climatology (0\%). Only 1 forecast month at lead 8 months lead reached the 16-20\% skill score level.

Using the composite neural network approach, for Miles with a lead time of 12 months, Table 5 shows that all 12 forecast months have a skill above climatology, falling in the range between $32 \%$ and $62 \%$. In contrast, examining the results POAMA grid region E for lead times of 6 and 8 months, there are 5 and 6 forecast months respectively where skill is below climatology (0\%), and only 1 forecast month where skill reaches the 16-20\% level.

For Deniliquin, all 12 forecast months showed a forecast skill above climatology using the composite model, with skill levels in the range $17 \%$ to $80 \%$. In contrast, examining the results 
Table 8: Forecast skill scores for monthly rainfall for three different grid regions produced by POAMA [10].

\begin{tabular}{|c|c|c|c|c|c|c|}
\hline \multirow[b]{2}{*}{ Lead (months) } & \multicolumn{6}{|c|}{ Skill relative to climatology $(\%)$} \\
\hline & -20 to 0 & 0 to 4 & 4 to 8 & 8 to 12 & 12 to 16 & 16 to 20 \\
\hline \multicolumn{7}{|c|}{ Grid region $\mathrm{H}$ (proximity to Bathurst) } \\
\hline 8 & 8 & 1 & 0 & 0 & 2 & 1 \\
\hline 6 & 7 & 2 & 1 & 1 & 1 & 0 \\
\hline 0 & 6 & 0 & 2 & 1 & 1 & 2 \\
\hline \multicolumn{7}{|c|}{ Grid region G (proximity to Deniliquin) } \\
\hline 8 & 9 & 2 & 1 & 0 & 0 & 0 \\
\hline 6 & 7 & 2 & 1 & 2 & 0 & 0 \\
\hline 0 & 4 & 4 & 1 & 3 & 0 & 0 \\
\hline \multicolumn{7}{|c|}{ Grid region E (proximity to Miles) } \\
\hline 8 & 5 & 2 & 2 & 2 & 0 & 1 \\
\hline 6 & 6 & 4 & 1 & 0 & 1 & 0 \\
\hline 0 & 2 & 2 & 5 & 2 & 1 & 0 \\
\hline
\end{tabular}

POAMA for grid region $\mathrm{G}$ for lead times of 6 and 8 months, there are 6 and 8 forecast months respectively where skill is below climatology $(0 \%)$, and no forecast months where skill reaches the 16-20\% level.

Shao and $\mathrm{Li}$ [9] reported results of a downscaling technique to produce monthly rainfall forecasts for 35 sites in the Murray Darling basin based on results from POAMA 1.5 over the period 1980-2006. The sites were concentrated in the region between Bathurst and Deniliquin. Detailed analysis of forecasts for individual sites was not reported, and forecast accuracy reported only as correlation coefficients. For lead times of 1,2 and 3 months the mean correlations between observed and downscaled monthly rainfall were: $0.381,0.287$ and 0.192 respectively. Correlations for individual months fell in the range between 0.1 and 0.6. This can be contrasted with correlation coefficients for the composite neural network approach in Table 3, where correlation coefficients fall in the range from 0.80 to 0.98 . These results suggest that the predictive skill of POAMA for monthly rainfall is very low, particularly at long lead times.

The demonstrated skill of GCMs in predicting seasonal or monthly rainfall is low for many regions of the world including India, the United States and Australia [37-40] often with little enhancement in forecast skill above climatology. A number of different approaches have been investigated to improve the skill of GCMs in predicting rainfall, including using weighted multi-model ensembles [41] and developing hybrid statistical models [42-44]. Post-processing of the output of the GCM POAMA using statistical models has been reported in attempting to improve skill of rainfall forecasts for Australia [39, 40].

Operational seasonal rainfall is currently produced by the BOM in Australia, use a combination of dynamical and statistical modeling. The dynamical model used is POAMA [40], while the statistical model makes use of the climate variables derived from POAMA [45]. 
Effectively, this amounts to using the GCM to forecast parameters, particularly concurrent large scale climate indices, such as sea surface temperatures, for the input statistical model. While this approach may have some merit, the question then arises as to whether the input parameters generated by the GCM might be generated more skilfully using a statistical model such as a neural network, rather than the GCM. Halide and Ridd [46] have suggested that, despite their complexity and the superior computational power of the super computers used to run them, GCMs are no better at forecasting parameters associated with ENSO than very simple statistical models. It has been shown [47] that forecasts of the Niño indices can be performed more skilfully by ANNs than by GCMs, and do not suffer from the limitations imposed by the "Spring Predictability Barrier" [48, 49].

In addition to the low skill in the operational seasonal rainfall [44] forecasts generated by the BOM, there are a number of other important issues regarding the form in which the forecasts are provided to the public. The operational seasonal forecasts are provided to the public only in a probabilistic, as opposed to a deterministic form. This means the information provided is only the probability that rainfall will be above the median value, rather than as a quantitative estimate of the rainfall to be anticipated. Surveys have shown that end-users of probabilistic forecasts, such as farmers, often find this concept difficult to interpret and apply in practical terms [50]. For example, a forecast that there is a probability of $60 \%$ that rainfall will be above median gives limited information about the amount of rainfall to anticipate the forecast is very limited because it does not differentiate between, for example, being $1 \%$ higher than the median or $400 \%$ higher than the median.

This shortcoming in probabilistic operational forecasts can be illustrated with a recent example, In May 2016 the BOM issued a 3 month rainfall forecast for Australia [51] indicating that there was a 70-80\% chance of above median rainfall over much of the Murray Darling Basin during the period June to August 2016. This would have delighted many farmers in the region, who would have enthusiastically planted their crops with the expectation of bumper harvests, following years of comparative drought and widespread depression in farming communities. In fact, record rainfalls in the three month forecast period in parts of the MDB caused extensive flooding, washing away about $30 \%$ winter crops such as wheat, barley and chickpeas. The New South Wales Department of Primary Industries (DPI) said it had been the third wettest winter on record [52]. The extreme rainfall resulted in extensive flooding leaving the town of Deniliquin devastated [53]. Although it can be argued the BOM operational seasonal forecast was "correct" it did not provide the information many end-users required to make appropriate decisions regarding crop management.

Other limitations on the operational forecasts are the comparatively long duration (seasonal as opposed to monthly) and the short forecast lead times (limited to the next three months). The ANN approach, in contrast, is very flexible in generating forecast with different forecast periods and lead times to suit the need of different end-users. Output from the GCM also corresponds to large geographical areas corresponding to grid areas of $250 \mathrm{~km} \times 250 \mathrm{~km}$, rather than more localised forecasts that are easily generated by the ANN approach.

\section{CONCLUSION}

This study builds on previous investigations that used ANNs for monthly rainfall forecasting with lead-times up to three months [19-21]. This study indicates that it is possible to make skilful monthly rainfall forecasts with a 12 month lead time for locations in the Murray Darling Basin. Our previous approach of forecasting has also been refined by examining the skill corresponding to the optimization of individual months, as well as the overall 
performance of multi-month optimization. In the majority of cases, forecast skill using the "single-month" optimisation is higher than the "all-month" optimisation. A composite forecast was constructed for each site by taking the best results from each method.

Comparisons of the predictive skill of the composite ANN model with rainfall forecasts derived from the GCM POAMA show that the ANN approach has significantly superior skill at the three locations in the Murray Darling Basin investigated. At the long lead time of 12 months the ANN approach has skill of $40 \%$ above that of climatology, whereas skill levels derived from forecasts using POAMA at long lead times are generally only comparable to climatology (Tables 4 and 7). There remains scope for future improvement, for example, through the introduction of other input parameters that have been associated with rainfall, such as blocking [16] and SAM [16, 34], although there may be an offsetting impact of increased numbers of attributes against shorter data sets due to more limited availability of historical values.

Despite intensive research efforts and heavy investment over the past two decades, the skill of operational rainfall forecasts reliant on the GCM POAMA remains low, and provides information in a format that is often not useful to the public. This study and others show that more skilful rainfall forecasts can be generated using ANNs, and these have flexibility in forecast periods and lead times. Recognising that GCMs perform poorly in prediction of rainfall, much of the recent efforts have been directed towards skill improvement by combining their output with statistical models. Although some improvement in skill has resulted, it may now be time to accept that the public would be better served by developing medium-term rainfall forecasts focussing on machine leaning and artificial intelligence technologies such as neural networks.

\section{ACKNOWLEDGEMENTS}

This research was funded by the B. Macfie Family Foundation. Chris Folland from the UK Met Office provided the values of IPO.

\section{REFERENCES}

[1] Australian Bureau of Statistics, Water and the Murray-Darling Basin - a statistical profile, 2008, available at http://www.abs.gov.au/ausstats/abs@.nsf/mf/4610.0.55.007

[2] Ashton, D. \& Oliver, M., Irrigation technology and water use on farms in the MurrayDarling Basin 2006-2007 to 2011-2012. ABARES Research Report 14.03, 2014.

[3] Gell, P., Mills, K. \& Grundell, R., Challenges to management of environment and agri culture: A legacy of climate and catchment change: the real challenge for wetland management. Hydrobiologia, 708(1), pp. 133-144, 2013. https://doi.org/10.1007/s10750-012-1163-4

[4] Gallant, A.J.E., Reeder, M.J., Risbey, J.S. \& Henessey, K.J., The characteristics of seasonal-scale droughts in Australia, 1911-2009. International Journal of Climatology, 33(7), pp. 1658-1672, 2013.

https://doi.org/10.1002/joc.3540

[5] Pook, M., Lisson, S., Risbey, J., Ummenhofer, C.C., McIntosh, P. \& Rebbeck, M., The autumn break for cropping in southeast Australia: trends, synoptic influences and impacts on wheat yield. International Journal of Climatology, 29, pp. 2012-2026, 2009. https://doi.org/10.1002/joc.1833

[6] Vizard, A.L., \& Anderson, G.A., The resolution and potential value of Australian seasonal rainfall forecasts based on the five phases of the Southern Oscillation Index. Crop \& Pasture Science, 60, pp. 230-239, 2009. https://doi.org/10.1071/CP08277 
[7] Hammer, G.L, Holzworth, D.P. \& Stone, R., The value of skill in seasonal climate forecasting to wheat crop management in a region with high climatic variability. Australian Journal of Agricultural Research, 47, pp. 717-737, 1996. https://doi.org/10.1071/AR9960717

[8] Ash, A., McIntosh, P., Cullen, B., Carberry, P. \& Stafford Smith, M., Constraints and opportunities in applying seasonal climate forecasts in agriculture. Australian Journal of Agricultural Research, 58, pp. 952-965, 2007. https://doi.org/10.1071/AR06188

[9] Shao, Q. \& Li, M., An improved statistical analogue downscaling procedure for seasonal precipitation forecast. Stochastic Environmental Research and Risk Assessment, 27, pp. 819-830, 2013. https://doi.org/10.1007/s00477-012-0610-0

[10] Hawthorne, S., Wang, Q.J., Schepen, A. \& Robertson, D., Effective use of general circulation model outputs for forecasting monthly rainfalls to long lead times. Water Resources Research, 49, pp. 5427-5436, 2013. https://doi.org/10.1002/wrcr.20453

[11] Drosdowsky, W. \& Chambers, L.E., Near-Global Sea Surface Temperature Anomalies as Predictors of Australian Seasonal Rainfall. Journal of Climate, 14, pp. 1677-1687, 2001. https://doi.org/10.1175/1520-0442(2001)014<1677:NACNGS>2.0.CO;2

[12] Fawcett, R.J.B. \& Stone, R.C., A comparison of two seasonal rainfall forecasting systems for Australia. Australian Meteorological and Oceanographic Journal, 60, pp. 15-24, 2010. https://doi.org/10.22499/2.6001.002

[13] Schepen, A., Wang, Q.J. \& Robertson, D.E., Seasonal forecasts of Australian rainfall through calibration and bridging of coupled GCM outputs. Monthly Weather Review, 142, pp. 1758-1777, 2014. https://doi.org/10.1175/MWR-D-13-00248.1

[14] Schepen, A., Wang, Q.J. \& Robertson, D., Evidence for using lagged climate indices to forecast Australian seasonal rainfall. Journal of Climate, 25, pp. 1230-1246, 2012. https://doi.org/10.1175/JCLI-D-11-00156.1

[15] Murphy, B.F. \& Ribbe, J., Variability of Southeastern Queensland Rainfall and climate indices international. Journal of Climatology, 24, pp. 703-721, 2004. https://doi.org/10.1002/joc.1018

[16] Risbey, J.S., Pook, M.J., Mcintosh, P.C., Wheeler, M.C. \& Hendon, H.H., On the remote drivers of rainfall variability in Australia. Monthly Weather Review, 137, pp. 3233-3253, 2009.

https://doi.org/10.1175/2009MWR2861.1

[17] Ramseyer, C.A. \& Mote, T.L., Atmospheric controls on Puerto Rico precipitation using artificial neural networks. Climate Dynamics, 47(7), pp. 2515-2526, 2016. https://doi.org/10.1007/s00382-016-2980-3

[18] Dahamsheha, A. \& Aksoy, H., Artificial neural network models for forecasting intermittent monthly precipitation in arid regions. Applied Meteorology, 16, pp. 325-337, 2009. https://doi.org/10.1002/met.127

[19] Nayak, D.R., Mahapatra, A. \& Mishra, P., A survey on rainfall prediction using artificial neural network. International Journal of Computer Applications, 72(16), pp. 32-40, 2013. https://doi.org/10.5120/12580-9217

[20] Abbot, J. \& Marohasy J., Input selection and optimisation for monthly rainfall forecasting in Queensland, Australia, using artificial neural networks. Atmospheric Research, 138, pp. 166-178, 2014. https://doi.org/10.1016/j.atmosres.2013.11.002 
[21] Abbot, J. \& Marohasy, J., The potential benefits of using artificial intelligence for monthly rainfall forecasting for the Bowen Basin, Queensland, Australia. Water Resources Management VII. WIT Transactions on Ecology and the Environment, 171, pp. 287-297, 2013.

https://doi.org/10.2495/WRM130261

[22] Abbot, J. \& Marohasy, J., Using lagged and forecast climate indices with artificial intelligence to predict monthly rainfall in the Brisbane Catchment, Queensland, Australia. International Journal of Sustainable Development and Planning, 10(1), pp. 29-41, 2015. https://doi.org/10.2495/SDP-V10-N1-29-41

[23] Abbot, J. \& Marohasy, J., Application of artificial neural networks to rainfall forecasting in Queensland, Australia. Advances in Atmospheric Sciences, 29(4), pp. 717-730, 2012. https://doi.org/10.1007/s00376-012-1259-9

[24] Vaze, J., Teng, J. \& Chiew, F.H.S., Assessment of GCM simulations of annual and seasonal rainfall and daily rainfall distribution across south-east Australia. Hydrological Processes, 25, pp. 1486-1497, 2011. https://doi.org/10.1002/hyp.7916

[25] Langford, S. \& Hendon, H.H., Improving reliability of coupled model forecasts of Australian Seasonal Rainfall. Monthly Weather Review, 141, pp. 728-741, 2013. https://doi.org/10.1175/MWR-D-11-00333.1

[26] Chowdhury, R.K. \& Beecham, S., Australian rainfall trends and their relation to the southern oscillation index. Hydrological Processes, 24, pp. 504-514, 2010. https://doi.org/10.1029/2007GL030854

[27] Power, S.B. \& Smith, I.M., Weakening of the walker circulation and apparent dominance of El Nino both reach record levels, but has ENSO really changed? Geophysical Research Letters, 34, L18702, 2007.

[28] Cai, W., Whetton, P.J. \& Pittock, A.B., Fluctuations of the relationship between ENSO and northeast Australian rainfall. Climate Dynamics 17, pp. 421-432, 2001. https://doi.org/10.1007/PL00013738

[29] Cai, W., et al., Asymmetry in ENSO teleconnection with regional rainfall, its multidecadal variability, and impact. Journal of Climate, 23, pp. 4944-4955, 2010. https://doi.org/10.1175/2010JCLI3501.1

[30] Power, S.B., et al., Interdecadal modulation of the impact of ENSO on Australia. Climate Dynamics, 15, pp. 319-324, 1999. https://doi.org/10.1007/s003820050284

[31] Power, S.B., et al., The predictability of interdecadal changes in ENSO activity and ENSO teleconnections. Journal of Climate, 19, pp. 4755-4771, 2006. https://doi.org/10.1175/JCLI3868.1

[32] Verdon, D.C. \& Franks, S.W., Long-term behaviour of ENSO: interactions with the PDO over the past 400 years inferred from paleoclimate records. Geophysical Research Letters, 33, L06712, 2006. https://doi.org/10.1029/2005GL025052

[33] Chiew, F.H.S., et al., El Nino/Southern oscillation and Australian Rain, streamflow and drought: links and potential for forecasting. Journal of Hydrology, 204, pp. 138-149, 1998. https://doi.org/10.1016/S0022-1694(97)00121-2

[34] Kirono, D.G.C., Chiew, F.H.S. \& Kent, D.M., Identification of best predictors for forecasting seasonal rainfall and runoff in Australia. Hydrological Processes, 24, pp. 1237$1247,2010$.

https://doi.org/10.1002/hyp.7585 
[35] Abbot, J. \& Marohasy, J., Forecasting of monthly rainfall in the Murray Darling Basin, Australia: Miles as a case study. River Basin Management VIII Book Series: WIT Transactions on Ecology and the Environment, 197, pp. 149-159, 2015. https://doi.org/10.2495/rm150141

[36] Mekanik, F., Imteaz, M.A., Gato-Trinidad, S. \& Elmahdi, A., Multiple regression and Artificial Neural Network for long-term rainfall forecasting using large scale climate modes. Journal of Hydrology, 503, pp. 11-21, 2013. https://doi.org/10.1016/j.jhydrol.2013.08.035

[37] Zimmerman, B.G., Vimont, D. J. \& Block, P. J., Utilizing the state of ENSO as a means for season-ahead predictor selection. Water Resources Research, 52(5), pp. 3761-3774, 2016. https://doi.org/10.1002/2015WR017644

[38] Tiwari, P.R., Kar, S.C., Mohanty, U.C., et al., Skill of precipitation prediction with GCMs over north India during winter season. International Journal of Climatology, 34(12), pp. 3440-3455, 2014. https://doi.org/10.1002/joc.3921

[39] Schepen, A. \& Wang, Q. J., Ensemble forecasts of monthly catchment rainfall out to long lead times by post-processing coupled general circulation model output. Journal of Hydrology, 519, pp. 2920-2931, 2014. https://doi.org/10.1016/j.jhydrol.2014.03.017

[40] Schepen, A. \& Wang, Q. J., Toward accurate and reliable forecasts of Australian seasonal rainfall by calibrating and merging multiple coupled GCMs. Monthly Weather Review, 141(12), pp. 4554-4563, 2013. https://doi.org/10.1175/MWR-D-12-00253.1

[41] Acharya, N., Chattopadhyay, S., Mohanty, U.C. \& Ghosh, K., Prediction of Indian summer monsoon rainfall: a weighted multi-model ensemble to enhance probabilistic forecast skills. Meteorological Applications, 21, pp. 724-732, 2014. https://doi.org/10.1002/met.1400

[42] Lang, X. \& Wang, H., Improving extraseasonal summer rainfall prediction by merging information from GCMs and observations. Weather and Forecasting, 25(4), pp. 1263-1274, 2010. https://doi.org/10.1175/2010WAF2222342.1

[43] Ying, L. \& Ke, F., Improve the prediction of summer precipitation in the Southeastern China by a hybrid statistical downscaling model. Meteorology and Atmospheric Physics, 117(3-4), pp. 121-134, 2012. https://doi.org/10.1007/s00703-012-0201-0

[44] Sun, J. \& Chen, H., A statistical downscaling scheme to improve global precipitation forecasting. Meteorology and Atmospheric Physics, 117(3), pp. 87-102, 2012. https://doi.org/10.1007/s00703-012-0195-7

[45] Wang, Q.J., Schepen, A. \& Robertson, D.E., Merging seasonal rainfall forecasts from multiple statistical models through Bayesian model averaging. Journal of Climate, 25(16), pp. 5524-5537, 2012. https://doi.org/10.1175/JCLI-D-11-00386.1

[46] Halide, H. \& Ridd, P., Complicated ENSO models do not significantly outperform very simple ENSO models. International Journal of Climatology, 28, pp. 219-233, 2008. https://doi.org/10.1002/joc.1519

[47] Abbot, J. \& Marohasy, J., Forecasting monthly rainfall in the Bowen Basin of Queensland, Australia, using neural networks with Niño Indices for El Niño-Southern Oscillation. Lecture Notes in Artificial Intelligence, 9992, pp. 88-100, 2016. 
[48] Duan, W. \& Zhang, R., Is model parameter error related to a significant spring predictability barrier for El Nino events? Results from a theoretical model. Advances in Atmospheric Science, 27(5), pp. 1003-1013, 2010. https://doi.org/10.1007/s00376-009-9166-4

[49] Yan, L. \& Yu, Y., The spring prediction barrier in ENSO hindcast experiments using the FGOALS-g model. Chinese Journal of Oceanology Limnology, 30(6), pp. 1093-1104, 2012. https://doi.org/10.1007/s00343-012-1271-z

[50] Coventry, W.L. \& Dalgleish, L.I., Farmers' accuracy interpreting seasonal climate forecast probability. International Journal of Climatology, 34(6), pp. 2097-2107, 2014. https://doi.org/10.1002/joc.3825

[51] http://www.bom.gov.au/jsp/sco/archive/index.jsp?map=rain\&outlook=probmedian \&p eriod $=$ season $1 \&$ year $=2016 \&$ month $=05 \&$ day $=26$

[52] Woodburn, J., Rain wipes out NSW crops in third wettest winter on record. ABC News, available at http://www.abc.net.au/news/2016-09-02/rain-wipes-out-nsw-crops-in-third-wettestwinter-on-record/7810722, September 2, 2016.

[53] Hoerr, K., NSW flooding leaves rural town of Deniliquin devastated, community set to feel impact for months. ABC News, available at http://www.abc.net.au/news/2016-10-16/nsw-floodingleaves-rural-town-of-deniliquin-devastated/7935136, October 16, 2016. 